\title{
A case of Klinefelter syndrome with hypersexual desire
}

\author{
Kingsley Okolie', Sumathy Perampalam²,3, Anthony Barker ${ }^{4}$ and Christopher J Nolan 2,3 \\ ${ }^{1}$ National Health Co-op, Australian Capital Territory (ACT), Canberra, Australia, 2Department of Endocrinology, \\ Canberra Hospital, Canberra, ACT, Australia, ${ }^{3}$ Australian National University Medical School, Canberra, ACT, \\ Australia, and ${ }^{4}$ Forensic Mental Health, Justice Health and Alcohol and Drug Services, Canberra, ACT, Australia
}

Correspondence should be addressed to K Okolie

Email

kingschinwe@yahoo.com

\section{Summary}

Klinefelter syndrome (KS) is a chromosomal disorder affecting males, with the typical karyotype of 47,XXY due to a supernumerary $X$ chromosome, which causes progressive testicular failure resulting in androgen deficiency and infertility. Despite it being the most common sex chromosomal disorder, its diagnosis is easily missed. In addition to its classical clinical features of tall stature, gynaecomastia, small testes, and symptoms and signs of hypogonadism including infertility, KS is also often associated with neurocognitive, behavioural and psychiatric disorders.

We present a 44-year-old man with KS who, despite having erectile dysfunction, paradoxically had increased libido. He used sildenafil to overcome his erectile dysfunction. Hypersexuality was manifested by very frequent masturbation, multiple sexual partners most of whom were casual, and a sexual offence conviction at the age of 17 years.

Discussion focuses on the frequent failure of clinicians to diagnose $\mathrm{KS}$, the neurocognitive, behavioural and psychiatric aspects of $\mathrm{KS}$, this unusual presentation of hypersexuality in a man with $\mathrm{KS}$, and the challenges of medical management of hypogonadism in a man with a history of a sexual offence.

\section{Learning points:}

- Klinefelter syndrome (KS) is common in men (about 1 in 600 males), but the diagnosis is very often missed.

- In addition to classic features of hypogonadism, patients with KS can often have associated neurocognitive, behavioural and/or psychiatric disorders.

- More awareness of the association between KS and difficulties related to verbal skills in boys could improve rates of early diagnosis and prevent longer-term psychosocial disability.

- Hypersexuality in the context of hypogonadism raises the possibility of sex steroid independent mechanistic pathways for libido.

- Testosterone replacement therapy in KS with hypersexuality should be undertaken with caution using a multidisciplinary team approach.

\section{Background}

Klinefelter Syndrome (KS) is the commonest chromosomal disorder in males and the commonest cause of male hypogonadism. It affects about 1 in 600 men, however the majority remain undiagnosed $(1,2)$. Classically, it is defined by the $47, \mathrm{XXY}$ karyotype, but variants exist (e.g. mosaics; 48,XXXY; 49,XXXY). It results from non-disjunction of the $\mathrm{X}$ chromosome, either in meiosis or mitosis. When it occurs in mitosis post-fertilization, a mosaic form $(46, \mathrm{XY} / 47, \mathrm{XXY})$ can occur accounting for about $10 \%$ of cases $(1,2)$.

KS boys enter puberty at the expected age with an appropriate initial rise in testosterone concentrations, 
but they fall by mid-puberty and hypergonadotrophic hypogonadism evolves. Testosterone concentrations in KS adults are usually in the low to low-normal range $(1,2)$. Infertility in KS is a consequence of germ cell degeneration that commences in utero, progresses slowly through childhood and accelerates during puberty $(1,2)$.

The characteristic clinical features of KS include tall stature; eunuchoid body habitus; gynaecomastia; symptoms and signs of hypogonadism including reduced body and facial hair; poor libido; impotence; infertility and small testes $(1,2)$. Concurrent cognitive, behavioural and/or psychiatric disorders can be present and in some cases be the presenting feature $(1,2,3)$. For example, boys with KS can present with learning difficulties, particularly due to delayed development of verbal skills, and/or with behavioural issues. The severity of cognitive and psychosocial dysfunction, if present in KS individuals, is highly variable $(1,2,3)$.

This case is of a man with a late diagnosis of KS, who had a history of learning and behavioural difficulties in his early years, and the unusual problem of increased rather than reduced libido.

\section{Case presentation}

A 44-year-old man, employed as a supermarket checkout operator, presented in 2014 with a long-term history of erectile dysfunction, lethargy, low mood and poor concentration. Because of daytime tiredness, he would often nap. He was not shaving regularly. Unexpectedly, he described markedly increased libido characterized by uncontrollable urges to masturbate and the need for frequent heterosexual encounters. With a partner, he had problems with erections and ejaculation, but reported benefit with the cGMP-specific phosphodiesterase type 5 inhibitor, sildenafil.

Other than symptoms of sexual dysfunction and low androgenization, he denied endocrine symptoms suggestive of pituitary, adrenal or thyroid disease.

His excessive libido had, and was still having, detrimental effects on his life. At age 17 years, he was convicted of a sexual offence against a minor (a 9 year old girl). Although he had been diagnosed a 'closet paedophile' by a psychologist, he denied any fantasies or urges to have sexual contact with children. From 20 years of age, he claimed that he had sex with more than 1800 partners, the majority ( $>95 \%$ ) being sex workers. He was attending regularly for sexually transmitted infection screening (more than 3 times in the last year) and had been treated for genital warts with imiquimod cream multiple times. He believed that he was afflicted by 'sexual addiction,' being unable to control his desires and behaviours.

He had a history of learning and behavioural difficulties. He stated that he used to play alone and was diagnosed with autism as a child for which he did not receive treatment. He had learning difficulties in school, particularly with language and more specifically comprehension. He started a degree programme in pharmacy, but found it very difficult and deferred his classes.

His other health problems include obesity, obstructive sleep apnoea awaiting continuous positive air pressure (CPAP) treatment, gout, intermittent sciatica and asthma. His weight had increased from 88 to $110 \mathrm{~kg}$ at the age of 30 years, at which time he stopped competitive dancing and exercising. After joining a life style modification programme, he managed to lose $10 \mathrm{~kg}$ of weight. His medications included budesonide/eformoterol Turbuhaler 200/6 $\mu \mathrm{g} /$ dose twice daily and salbutamol inhaler $100 \mu \mathrm{g} /$ dose as a reliever for asthma; pregabalin $300 \mathrm{mg}$ oral daily for sciatica; intermittent non-steroidal anti-inflammatory agents for gout; and sildenafil orally for impotence.

He was living alone and denied ever having a longterm partner. He used alcohol only occasionally, but denied ever being a smoker or using recreational drugs. He denied use of 'black market' testosterone preparations. He was not aware of a family history of mental illness, chromosomal disorders or other endocrine conditions.

On examination, his weight was $100.1 \mathrm{~kg}$, height was $1.84 \mathrm{~m}$ and body mass index was $29.6 \mathrm{~kg} / \mathrm{m}^{2}$. His blood pressure was $118 / 70 \mathrm{mmHg}$ sitting and $106 / 69 \mathrm{mmHg}$, standing. He was centrally obese without an obvious eunuchoid body habitus and his muscle bulk was poor. He had gynaecomastia and some non-pigmented abdominal stretch marks. Body hair was sparse on his face, torso and axillae, however pubic hair was present. His testes were small $(<5 \mathrm{~mL})$. He had a protruding jaw, without orbital ridging or spade-like hands, and his visual fields were normal. His cardiovascular and respiratory examinations were unremarkable.

\section{Investigation}

His morning testosterone was reduced at $6.6 \mathrm{nmol} / \mathrm{L}$ and $6.3 \mathrm{nmol} / \mathrm{L}$ on repeat (7.0-31.0) and his luteinizing hormone (LH) and follicle-stimulating hormone (FSH) were both elevated, 20U/L (<12) and 40U/L (<12) respectively, consistent with hypergonadotrophic hypogonadism. 
Sex hormone binding globulin was $25.1 \mathrm{nmol} / \mathrm{L}$ (14.548.4). Levels of prolactin, adrenocorticotropic hormone, growth hormone and insulin-like growth factor 1 were normal. Free thyroxine $\left(\mathrm{T}_{4}\right)$ was borderline low on two occasions, 9.7 and $9.2 \mathrm{pmol} / \mathrm{L}(10.7-17.0)$, but free triiodothyronine $\left(\mathrm{T}_{3}\right)$ and thyroid-stimulating hormone (TSH) levels were in the normal range. MRI of the pituitary was normal, such that secondary hypothyroidism or other pituitary conditions were thought unlikely.

Cytogenetic examination of 80 cells showed a male karyotype with an additional X chromosome (47, XXY) in 75 cells, 2 additional X chromosomes (48, XXXY) in one cell, and $46, \mathrm{XY}$ in 4 cells. The finding was diagnostic of mosaic KS. Fluorescent in situ hybridization (FISH) investigation, with probes specific for the centromeric regions of chromosomes $\mathrm{X}, \mathrm{Y}$ and 18, showed signal patterns consistent with the 3 cell lines observed in the conventional karyotype.

His bone densitometry revealed osteopenia. His intact parathyroid hormone level was $8.2 \mathrm{pmol} / \mathrm{L}$ (1.6-7.2), but his serum calcium and 25-hydroxy-vitamin D3 levels were normal. Fasting plasma glucose was $6.2 \mathrm{mmol} / \mathrm{L}$, raising the possibility of impaired glucose tolerance. His lipid profile was within acceptable limits. Other haematology and biochemistry investigations, including renal and liver function tests, were normal.

\section{Treatment}

It was felt that testosterone therapy was indicated for his presenting symptoms of lethargy, low mood and poor concentration and for the prevention of osteoporosis; however there were obvious concerns for sexual offence recidivism. For this reason, a multidisciplinary approach was taken involving the patient's general practitioner, a clinical psychologist, an endocrinologist and a forensic psychiatrist. The psychologist believed the risk of dysfunctional sexual behaviour was low and a decision was cautiously made to commence him on a low dose of short-acting testosterone therapy. He was commenced on testosterone $2 \%$ solution (Axiron) topically at a dose of $30 \mathrm{mg}$ per day. The usual initial dose is $60 \mathrm{mg}$ per day. The patient was given instruction to stop therapy if he experienced adverse effects. He returned a few weeks later and reported increased mood swings without change in libido or sexual function. He was reassured that his mood swings may have been caused by the initial rise in testosterone levels and he was encouraged to continue with therapy. He was switched to a low-dose, short-acting testosterone transdermal patch, $2.5 \mathrm{mg}$ daily (Androderm).
After another 8 weeks, he reported improved mood and energy, and the need for more frequent shaving. His testosterone was $8.6 \mathrm{nmol} / \mathrm{L}$.

He was eventually assessed by a forensic psychiatrist. It was his opinion that the patient did not elicit evidence of paraphilia and the risk of him committing another sexual offence was low. He concluded that it was safe to treat him with testosterone. He did not make an official mental health diagnosis. The psychiatrist, however, recommended therapy with the selective serotonin reuptake inhibitor (SSRI) fluoxetine to improve his mood and to utilize its recognized adverse effect of inhibiting libido to reduce his hypersexual desire. The patient declined fluoxetine, but agreed to ongoing psychotherapy with the psychologist.

\section{Discussion}

This case illustrates the challenges of KS diagnosis and highlights the importance of recognising the neurocognitive, behavioural and psychiatric aspects of this condition. Hypersexuality in a man with KS is very unusual and is discussed in relation to the available literature. Also, the challenges of ethical medical management of hypogonadism in a man with a history of a sexual offense are addressed.

The diagnosis of $\mathrm{KS}$ is missed in up to $90 \%$ of adolescents and $75 \%$ of adults (1). Intrauterine diagnosis rates are likely increasing with more frequent use of prenatal genetic testing, but still only in a minority of pregnancies. It is clear that health professional's increased awareness of KS and its clinical features is required. Diagnosis in adolescents is difficult, as they usually enter puberty at the normal time and it is not until later in teenage years that the clinical features of hypogonadism and testicular atrophy become evident. A lesson from this case is to think of this possibility in boys and adolescents who have learning difficulties or display abnormal psychosocial characteristics $(1,2)$.

This patient did emphasize the difficulty he had with comprehension at school. In neuropsychological testing KS subjects consistently perform poorly in verbal skills $(1,2)$. Behavioural and psychiatric disorders, including autism, attention deficit hyperactivity disorder, bipolar affective disorders and schizophrenia have been believed to be overrepresented in KS individuals; however the evidence base indicative of increased major psychiatric illness in KS is poor $(1,2,3,4,5)$. The learning difficulties and autism features present early, suggesting that they are independent of testosterone deficiency. It is unclear if early 
testosterone treatment can have an ameliorating effect on neurocognitive or behavioural aspects if commenced early in puberty (2).

The patient described here displayed anti-social behaviour once at the age of 17 and it involved a sexual offence involving a minor. Anti-social personality disorders resulting in passive-aggressive tendencies, criminal behaviours and interestingly paraphilia have been linked to $\mathrm{KS}$, but this has been questioned due to poor-quality evidence $(2,3,4,5)$. However, KS is a condition classically associated with low libido due to hypogonadism (6). Hence the hypersexual desire in this patient is very unusual. Sinha et al., described a 22 -yearold KS patient with similar issues (7). Hypersexual desires in this other man clearly interfered with his social and academic functioning, which interestingly was lessened by sertraline (an SSRI) therapy (7). Both cases meet Kafka's criteria for hypersexual desire - a pattern of repetitive and strong sexual urge, desires and behaviours that lead to clinically significant distress, adverse consequences or impairment of function (8).

These two cases of high libido on the background of hypogonadism point to mechanisms independent of the well-known effects of androgens in determining libido. The dopamine and acetylcholine signalling pathways within the brain may have a role. Hypersexual drive has been described as an adverse effect of dopamine agonists used for treating Parkinson's disease and acetylcholinesterase inhibitors used in the treatment of dementia (9).

This case raises the ethical issues of prescribing testosterone therapy in patients with prior sexual convictions. Studer et al. linked sexual offence recidivism to testosterone levels. In 501 convicted adult male sex offenders, it was found that men with higher testosterone levels tended to have committed the most invasive sexual crimes and were more likely to re-offend (10). It is for these reasons that a multidisciplinary approach is necessary, bringing together essential skills of a psychologist, psychiatrist and endocrinologist. Although testosterone offers benefits of physical well-being, the risk of precipitating a vulnerable individual towards criminal behaviour must be evaluated at an individual level. Careful introduction of testosterone therapy at low doses with an easily reversible delivery method, such as the topical agents used in this case, is preferable to long-acting intramuscular methods (testosterone undeconoate). Regular follow-up to ensure treatment is not causing adverse effects needs to be included in the management plan. It was disappointing that this man declined therapy with an SSRI agent.

In conclusion, KS adults have both significant physical symptoms of hypogonadism and neurocognitive, psychosocial and behavioural problems that should be managed together, with multi-disciplinary input. More awareness of the condition with increased efforts to diagnose it earlier is necessary to improve outcomes.

Declaration of interest

The authors declare that there is no conflict of interest that could be perceived as prejudicing the impartiality of this work.

\section{Funding}

This work did not receive any specific grant from any funding agency in the public, commercial or not-for-profit sector.

\section{Patient consent}

A written informed consent has been obtained from the patient, and the consent form is attached.

\section{Author contribution statement}

$\mathrm{Dr}$ Kingsley Okolie is a general practitioner, he was involved in the management of the patient and wrote the initial draft of this report. Dr Sumathy Perampalam is an endocrinologist, she was involved in the management of the patient and contributed to writing this report. Dr Anthony Barker is a forensic psychiatrist, he reviewed the patient and contributed to writing this report. Prof Christopher Nolan assisted Dr Okolie in researching the topic and in writing the report.

\section{References}

1 Groth KA, Skakkebaek A, Host C, Gravholt CH \& Bojesen A 2013 Clinical review: Klinefelter syndrome - a clinical update. Journal of Clinical Endocrinology and Metabolism 98 20-30. (doi:10.1210/jc.2012-2382)

2 Bonomi M, Rochira V, Pasquali D, Balercia G, Jannini EA, Ferlin A \& Klinefelter Italia NG 2017 Klinefelter syndrome (KS): genetics, clinical phenotype and hypogonadism. Journal Endocrinolology Investigation $\mathbf{4 0}$ 123-134. (doi:10.1007/s40618-016-0541-6)

3 Hong DS \& Reiss AI 2014 Cognitive and neurological aspects of sex chromosome aneuploidies. Lancet Neurolology 13 306-318. (doi:10.1016/S1474-4422(13)70302-8)

4 Cederlof M, Ohlsson Gotby A, Larsson H, Serlachius E, Boman M, Langstrom N, Landen M \& Lichtenstein P 2014 Klinefelter syndrome and risk of psychosis, autism and ADHD. Journal of Psychiatric Research 48 128-130. (doi:10.1016/j.jpsychires.2013.10.001)

5 Annagur BB \& Kandeger A 2014 Case of Klinefelter's syndrome in an 18-year-old male presented with antisocial personality disorder. Journal of Psychiatry and Neurological Sciences $\mathbf{2 7}$ 254-255. (doi:10.5350/dajpn2014270309) 
6 Corona G, Petrone L, Paggi F, Lotti F, Boddi V, Fisher A, Vignozzi L, Balercia G, Sforza A, Forti G, et al. 2010 Sexual dysfunction in subjects with Klinefelter's syndrome. International Journal of Andrology 33 574-580.

7 Sinha P, Jnanaprakasan PP \& Andrade C 2012 Hyperactive sexual desire in Klinefelter Syndrome: treatment with sertraline. Psychiatry and Clinical Neurosciences 66 533. (doi:10.1111/j.1440-1819. 2012.02375.x)
8 Kafka MP 2010 Hypersexual disorder: a proposed diagnosis for DSM-V. Archives of Sexual Behavior 39 377-400. (doi:10.1007/s10508-009-9574-7)

9 Segrec N, Zaman R \& Pregelj P 2016 Increased libido associated with donepezil treatment: a case report. Psychogeriatrics 16 70-72. (doi:10.1111/psyg.12113)

10 Studer LH, Aylwin AS \& Reddon JR 2005 Testosterone, sexual offense recidivism, and treatment effect among adult male sex offenders. Sex Abuse 17 171-81. (doi:10.1177/107906320501700207)

Received in final form 22 June 2017

Accepted 10 July 2017 